Draft Version November 3, 2018

Preprint typeset using $\mathrm{LAT}_{\mathrm{E}} \mathrm{X}$ style emulateapj v. 11/26/03

\title{
THE COSMOLOGICAL CONSEQUENCE OF AN OBSCURED AGN POPULATION ON THE RADIATION EFFICIENCY
}

\author{
Alejo Martínez-Sansigre \\ Max-Planck-Institut für Astronomie, Königstuhl 17, D-69117 Heidelberg, Germany; ams@mpia.de
}

ANDREW M. TAYLOR

Max-Planck-Institut für Kernphysik, Postfach 103980, D-69029 Heidelberg, Germany; andrew.taylor@mpi-hd.mpg.de Draft version November 3, 2018

\begin{abstract}
In light of recent indications for a large population of obscured active galactic nuclei (AGNs), we revisit the mean radiation efficiency from accretion onto supermassive black holes (SMBHs), $\langle\epsilon\rangle$, applying a bayesian approach. We use the integrated comoving energy density emitted by AGNs and compare it to the mass density of local SMBHs. When considering only optically-selected unobscured AGNs, we derive $\log _{10}[\langle\epsilon\rangle]=-1.77_{-0.11}^{+0.16}$ or $\langle\epsilon\rangle=1.7_{-0.4}^{+0.8} \%$. Using the AGNs selected using hard X-rays, which include unabsorbed and Compton-thin absorbed AGNs, we find $\log _{10}[\langle\epsilon\rangle]=-1.20_{-0.10}^{+0.15}$ or $\langle\epsilon\rangle=6.4_{-1.3}^{+2.6} \%$. Using optically-selected AGNs, and correcting for the obscured population, we inferr $\log _{10}[\langle\epsilon\rangle]=-$ $1.17_{-0.08}^{+0.11}$ or $\langle\epsilon\rangle=6.7_{-1.1}^{+1.9} \%$, which we consider our best estimate. We also repeat this calculation for intrinsically luminous AGNs $\left(M_{\mathrm{B}}<-23\right.$, quasars), comparing to the SMBH mass density in local elliptical galaxies, and find $\log _{10}[\langle\epsilon\rangle]=-1.27_{-0.11}^{+0.15}$ or $\langle\epsilon\rangle=5.4_{-1.2}^{+2.2} \%$. We discuss how our results can be used to estimate the mean spin of accreting SMBHs, both assuming the standard thin-disk model of accretion onto black holes and also comparing to more recent simulations. Our results can rule out maximally rotating SMBHs $\left(\langle a\rangle=0.998 \frac{G m_{\bullet}}{c^{2}}\right)$ at the $\geq 98 \%$ confidence level, as well as high rotation values $\left(\langle a\rangle \geq 0.9 \frac{G m \bullet \bullet}{c^{2}}\right)$ with $\geq 92 \%$ confidence. Our preferred values of $\langle a\rangle$ are $\sim 0.25-0.60 \frac{G m \bullet}{c^{2}}$, although even these might be overestimated. Hence, we find that on average, SMBHs are not rapidly spinning during accretion. Finally, using an independent measurement of Eddington ratios, we estimate the SMBH $e$-folding time for the brightest AGNs (quasars) to be $\langle\tau\rangle=100_{-60}^{+151} \mathrm{Myr}$.

Subject headings: galaxies : active-galaxies: nuclei-quasars: general-black hole physics - cosmology: miscellaneous -
\end{abstract}

\section{INTRODUCTION}

Active galactic nuclei (AGNs) are believed to be powered by accretion onto supermassive black holes (SMBHs), and are expected to leave behind dormant SMBHs (Salpeter 1964; Lynden-Bell 1969; Rees 1984). The observed energy per comoving volume radiated by AGNs, the density of local SMBHs and the radiation efficiency of accreting SMBHs can all be related, using conservation of energy, in an elegant argument proposed by Soltan (1982).

The original form of the "Soltan argument" estimated the density of relic SMBHs from the distribution of observed quasar fluxes (magnitudes) and making an educated guess for the efficiency. Nowadays, SMBHs are believed to reside at the centres of galaxies, and estimates of the SMBHs masses are possible (e.g. Kormendv \& Richstone 1995; Eckart \& Genzel 1996; Magorrian et al. 1998). More accurate measurements of the AGN luminosity functions (LFs) exist, meaning that the least constrained parameter is the radiation efficiency. Using variations of the Soltan argument, the efficiency can be estimated from the comparison between the estimated total radiated energy from AGNs with the local SMBH (e.g. Elvis et al. 2002; Yu \& Tremaine 2002; Marconi et al. 2004; Shankar et al. 2004).

The discovery of a significant population of obscured
AGNs at a range of redshifts (see Section 4) has important consequences for the global energetics of quasars and accretion onto SMBHs, given that the total energy output from the obscured population is comparable to that of the unobscured population (if not greater).

In this article, we address the issue of how this population can have a significant effect on the mean radiation efficiency from accretion onto SMBHs. We apply a bayesian inference technique to derive posterior probability distribution functions (PDFs) for the mean radiation efficiency of SMBHs, $\langle\epsilon\rangle$.

In Section 2 we outline how the total radiated energy density and the local SMBHs density can be used to estimate $\langle\epsilon\rangle$. In Section 3 we discuss the relative contributions of AGNs at different redshifts to the total radiated energy density. Section 4 summarises the current knowledge on the obscured AGN population. In Section 5 we explain in detail how we inferr $\langle\epsilon\rangle$, using a bayesian approach, and how we combine different estimates of $\langle\epsilon\rangle$. Section 6 discusses how the mean SMBH spin during periods of accretion can be estimated from $\langle\epsilon\rangle$. Finally, in Section 7 we estimate the $e$-folding time for quasars, and in Section 8 we summarise our results.

Throughout this article we refer to powerful AGNs as quasars where, for unobscured AGNs, the dividing line is generally taken at $M_{\mathrm{B}}=-23\left(L_{\nu \mathrm{B}}=7 \times 10^{22} \mathrm{~W} \mathrm{~Hz}^{-1}\right)$. We assume a $\Lambda \mathrm{CDM}$ cosmology with the following pa- 
rameters: $h=H_{0} /\left(100 \mathrm{~km} \mathrm{~s}^{-1} \mathrm{Mpc}^{-1}\right)=0.7 ; \Omega_{\mathrm{m}}=0.3$; $\Omega_{\Lambda}=0.7$.

\section{THE EFFICIENCY OF ACCRETION ONTO SMBHS}

We outline here the main steps of the Sołtan argument, in the form that we will use to inferr the mean radiation efficiency onto SMBHs, $\langle\epsilon\rangle$.

The total (comoving) energy density radiated by AGNs can be related to the integrated local SMBH density, $\rho_{\bullet}$, loc, using three assumptions: i) that SMBHs grew all their mass by accretion, thus neglecting mergers between black holes (or rather taking the simplifying assumption that mergers conserve SMBH mass) ii) that the mean efficiency $\langle\epsilon\rangle$ is constant with luminosity and over the Hubble time and iii) that AGNs are the manifestation of these periods of growth by accretion.

For an individual SMBH, accretion with radiation efficiency $\epsilon$ will lead to a bolometric energy:

$$
E_{\mathrm{bol}}=\int \epsilon \frac{\mathrm{d} m}{\mathrm{~d} t} c^{2} \mathrm{~d} t
$$

while a relic SMBH will be left behind, with mass,

$$
m_{\bullet, \text { acc }}=\int(1-\epsilon) \frac{\mathrm{d} m}{\mathrm{~d} t} \mathrm{~d} t .
$$

The radiated energy no longer contributes to the accreted mass energy, which the distant observer will see as the SMBH mass, hence the $(1-\epsilon)$ term. Rather than tracking individual sources, we are interested in the relic SMBH mass density $\left(\rho_{\bullet}\right.$, acc $)$ inferred from the summed comoving energy density emitted by AGNs over the age of the Universe, which can be estimated from the AGN LF. Converting to observable quantities, we obtain:

$$
\rho_{\bullet}, \text { acc }=\frac{(1-\langle\epsilon\rangle)}{\langle\epsilon\rangle c^{2}} \int \frac{\mathrm{d} t}{\mathrm{~d} z} \mathrm{~d} z \int C_{\nu} \nu L_{\nu} \phi\left(L_{\nu}, z\right) \mathrm{d} L_{\nu},
$$

where $\phi\left(L_{\nu}, z\right)$ is the LF for all AGNs, $C_{\nu}$ is the bolometric correction and $\langle\epsilon\rangle$ is the mean efficiency. The bolometric correction (defined so $L_{\mathrm{bol}}=C_{\nu} \nu L_{\nu}$ ) is necessary to convert from the monochromatic LF to the bolometric luminosity density.

If only an unobscured AGN LF is available, one can instead correct for the missing obscured AGNs:

$\rho_{\bullet, \text { acc }}=\frac{(1-\langle\epsilon\rangle)}{\langle\epsilon\rangle c^{2}}(1+\langle R\rangle) \int \frac{\mathrm{d} t}{\mathrm{~d} z} \mathrm{~d} z \int C_{\nu} \nu L_{\nu} \phi_{\mathrm{u}}\left(L_{\nu}, z\right) \mathrm{d} L_{\nu}$.

Here $\phi_{\mathrm{u}}\left(L_{\nu}, z\right)$ is the unobscured AGN LF. $\langle R\rangle$ is the mean ratio of obscured to unobscured AGNs so the term $(1+\langle R\rangle)$ is a parametrised way of accounting for the obscured population. Estimates exist for the parameters $C_{\nu}$ and $\langle R\rangle$. As mentioned earlier, the least constrained parameter is $\langle\epsilon\rangle$, so we treat it here as a free parameter.

\section{RADIATED ENERGY DENSITY}

A crucial quantity for the estimation of $\langle\epsilon\rangle$ is the total energy density radiated by AGNs, $U$ (the timeintegrated luminosity density). The AGN population shows a strong evolution so that the contribution of the low-redshift AGNs $(z \lesssim 0.3)$ to the energy density is very modest.

Amongst optically-selected AGNs, the luminosity density of AGNs strongly increases with redshift, with a peak around $z \sim 2-2.5$ (e.g. Wolf et al. 2003), and for the entire AGN population over cosmic time, $U$ is dominated by the AGNs at $z=1-3$ (particularly those around $L^{\star}$ ) with the lower-redshift AGNs contributing a smaller fraction.

This is illustrated in the left panel of Figure 1, which shows the cumulative energy density $U(z)$ [relative to $U(z=0)$ ] for several optical LFs and one hard X-ray LF. For the optical surveys, all defined in the B-band (centred on $4400 \AA$, or $2.8 \mathrm{eV}), U(z)$ is defined as:

$$
U_{\mathrm{B}}(z)=\int_{z^{\prime}=5}^{z^{\prime}=z} \frac{\mathrm{d} t}{\mathrm{~d} z^{\prime}} \mathrm{d} z^{\prime} \int C_{\mathrm{B}} \nu_{\mathrm{B}} L_{\nu \mathrm{B}} \phi_{\mathrm{B}}\left(L_{\nu \mathrm{B}}, z^{\prime}\right) \mathrm{d} L_{\nu \mathrm{B}} .
$$

where $h_{\mathrm{p}} \nu_{\mathrm{B}}=2.8 \mathrm{eV}$ ( $h_{\mathrm{p}}$ is Planck's constant). $L_{\nu \mathrm{B}}, \phi_{\mathrm{B}}$ and $C_{\mathrm{B}}$ are, respectively, the luminosity density, LF and bolometric correction at the B-band. Plotting $U(z) / U(0)$ removes the dependence on the bolometric correction and shows the relative contribution at different epochs.

As can be seen in the left panel of Figure 1 for optically-selected AGNs, approximately $70 \%$ of $U(z=0)$ is radiated between $z=1$ and 3 , with $20 \%$ radiated at $z<1$ and $10 \%$ at $z>3$.

However, similar studies undertaken at hard X-ray energies (in the $2-10 \mathrm{keV}$ band) have shown that the lowerluminosity AGNs evolve differently to high-luminosity AGNs, with their activity peaking at lower redshifts (e.g. Ueda et al. 2003). Again, we illustrate the effect by plotting $U(z)$ (Figure 1, right panel), with $U(z)$ defined as:

$$
U_{\mathrm{X}}(z)=\int_{z^{\prime}=5}^{z^{\prime}=z} \frac{\mathrm{d} t}{\mathrm{~d} z^{\prime}} \mathrm{d} z^{\prime} \int C_{\mathrm{X}} L_{\mathrm{X}} \phi_{\mathrm{X}}\left(L_{\mathrm{X}}, z^{\prime}\right) \mathrm{d} L_{\mathrm{X}}
$$

where $L_{\mathrm{X}}, \phi_{\mathrm{X}}$ and $C_{\mathrm{X}}$ are the hard X-ray luminosity, $\mathrm{LF}$ and bolometric correction, but note that here $L_{\mathrm{bol}}=$ $C_{\mathrm{X}} L_{\mathrm{X}}$.

From the right panel of Figure 1 we can see that only about $50 \%$ of $U$ was radiated between $z=1$ and 3 , with $\sim 45 \%$ radiated at $z<1$ and $\sim 5 \%$ at $z>3$.

Thus, from optically-selected LFs one expects the AGNs at $1 \leq z \leq 3$ to dominate, although the hard $\mathrm{X}$-ray LF indicates that the contribution of $z<1$ AGNs is similarly important and should not be overlooked.

\section{DEMOGRAPHY OF OBSCURED AGNS}

Obscured AGNs are believed to be accreting SMBHs where, between X-ray and near-infrared wavelengths, gas and dust block the line of sight to the central region. The strong continuum from the accretion disk and the broad emission lines suffer from heavy extinction by dust, while the narrow emission lines may sometimes be visible, depending on the obscuring geometry. Photoelectric absorption by the intervening gas and dust will supress the soft X-ray emission $(\$ 5 \mathrm{keV})$. If the column density is large enough to be optically-thick to Compton scattering $^{1}$, then even the hard X-rays $(\gtrsim 5 \mathrm{keV})$ will be heavily affected.

The obscured AGNs are indistinguishable from galaxies in optical and near-infrared imaging surveys, which has made their identification significantly harder than that of their unobscured counterparts. There are mainly

1 A source is Compton-thick if the absorbing column density, $N_{\mathrm{H}}$, is $\geq 1 / \sigma_{T}=1.5 \times 10^{28} \mathrm{~m}^{-2}$, where $\sigma_{T}$ is the Thomson electron scattering cross-section. X-ray absorbed, Compton-thin sources have $10^{26} \mathrm{~m}^{-2} \lesssim N_{\mathrm{H}} \lesssim 10^{28} \mathrm{~m}^{-2}$, and X-ray unabsorbed sources have $N_{\mathrm{H}} \lesssim 10^{26} \mathrm{~m}^{-2}$ 
four methods for looking for these objects, which we summarise in chronological order, and we concentrate here on the most recent results.

Radio selection (wavelength $\gtrsim 6 \mathrm{~cm}$, energy $\lesssim 20 \mu \mathrm{eV}$ ). Dust and gas are transparent to moderately highfrequency radio waves, so radio-loud obscured AGNs can be readily identified, in the form of narrow-line radio galaxies. Amongst the radio-loud population, the obscured to unobscured ratio is found to be $\sim 1: 1$ (Willott et al. 2000). However, the fraction of unobscured AGNs that are radio loud is small $(\sim 10 \%$, e.g. Ivezić et al. 2002), so that the narrow-line radio galaxies are expected to represent a similarly small fraction of the obscured AGN population (e.g. Zakamska et al. 2004).

Selection at hard X-ray energies $(\sim 2-10 \mathrm{keV})$. This method is sensitive to the Compton-thin absorbed AGN population, and it has identified large numbers of sources in deep X-ray surveys (see Brandt \& Hasinger 2005, for a review). Gilli et al. (2007) find the ratio of Comptonthin absorbed to unabsorbed AGNs to vary from $\sim 1: 1$ at the high luminosity end $\left(L_{\mathrm{X}} \sim 10^{12} \mathrm{~L}_{\odot}\right)$ to $\sim 4: 1$ at the low luminosity end $\left(L_{\mathrm{X}} \sim 10^{8} \mathrm{~L}_{\odot}\right)$. Using the hard X-ray background (at energies $\sim 3-100 \mathrm{keV}$ ) as an extra constraint, these authors find that the Compton-thick and Compton-thin absorbed populations are likely to have similar numbers (i.e $~ 2: 1$ at high luminosities and up to $\sim 8: 1$ at low luminosities).

Selection using optical spectroscopy (wavelengths $\sim 3500-7500 \AA$, energies $\sim 1.5-3.5 \mathrm{eV}$ ). If the geometry of the dust is such that the narrow emission lines are not obscured, these are detectable via optical spectroscopy. Using the spectroscopic database from the Sloan Digital Sky Survey, Zakamska et al. (2003) were able to select large numbers of obscured luminous AGNs (obscured quasars). Using the [O III] $5007 \AA$ line strength as a isotropic proxy for the intrinsic AGN luminosity, the ratio of spectroscopically-selected obscured quasars to unobscured quasars is $\sim 1.2-1.5: 1$ (Reves et al. 2008,

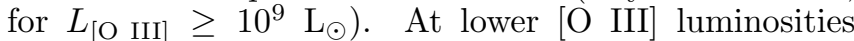
$\left(L_{[\mathrm{O} \text { III }]} \sim 10^{7} \mathrm{~L}_{\odot}\right)$ Simpson $(2005)$ estimate the obscured to unobscured ratio to be $\sim 4: 1$.

Mid-infrared selection (wavelengths $\sim 3-30 \mu \mathrm{m}$, energies $0.04-0.4 \mathrm{eV}$ ). The extinction due to dust becomes very small at these wavelengths, so mid-infrared selection should be sensitive to the radio-quiet population, including obscured AGNs showing no narrow lines. Recent work has suggested it is also sensitive to the Compton-thick population (e.g. Polletta et al. 2006; Martínez-Sansigre et al. 2007). However, care must be taken to separate starburst contaminants from real AGNs, since these star-forming galaxies also show bright mid-infrared emission. Combining mid-infrared and radio criteria, Martínez-Sansigre et al. (2008) found a $\sim 2: 1$ ratio at the high-luminosity end $\left(\nu L_{\nu 8 \mu \mathrm{m}} \gtrsim 10^{12} \mathrm{~L}_{\odot}\right)$, while Donley et al. (2005) found a $\sim 4: 1$ ratio amongst lower luminosity $\operatorname{AGNs}\left(\nu L_{\nu} 24 \mu \mathrm{m} \sim 10^{10} \mathrm{~L}_{\odot}\right)$.

Based on mid-infrared selection with no radio criteria, Lacy et al. (2007) found a $\sim 2: 1$ ratio amongst bright AGNs (with a median value $\nu L_{\nu} 5 \mu \mathrm{m} \sim 5 \times 10^{11} \mathrm{~L}_{\odot}$ ). Using a power-law criterion, Alonso-Herrero et al. (2006) also found a $\sim 2: 1$ ratio amongst powerful AGNs $(70 \%$ of their sources having $L_{\mathrm{IR}} \geq 10^{12} \mathrm{~L}_{\odot}$ ) while Donley et al. (2007) find a 4:1 ratio for less luminous sources (with typically $L_{\mathrm{X}} \lesssim 5 \times 10^{9} \mathrm{~L}_{\odot}$ or expected total infrared luminosities $L_{\mathrm{IR}} \lesssim 10^{11} \mathrm{~L}_{\odot}$ ) but only 2:1 if the sources are required to be $\mathrm{X}$-ray detected.

A possible picture fitting these observations is the following: amongst luminous AGNs (quasars), the obscured to unobscured ratio seems to be $\sim 2: 1$, with about half of the obscured quasars being Comptonthick (Gilli et al. 2007; Martínez-Sansigre et al. 2007), and about half showing no narrow emission lines (Martínez-Sansigre et al. 2008, which explains the $\sim 1: 1$ ratio found from spectroscopically-selected samples by Reyes et al., 2008). Amongst radio-loud quasars, the obscured to unobscured ratio is $\sim 1: 1$ with almost all obscured quasars (narrow-line radio galaxies) showing narrow emission lines. An explanation for this is that the lack of narrow lines is due to kpc-scale dust (e.g. Martínez-Sansigre et al. 2006; Rigby et al. 2006) and radio jets are efficient at clearing dust (Baker et al. 2002; Willott et al. 2002), so the fraction of radio galaxies obscured by kpc-scale dust is small.

Amongst the low-luminosity AGNs, from X-ray, optical spectroscopy and mid-infrared studies the obscured to unobscured ratio seems to be $\sim 4: 1$, consistent with the ratio inferred in the local Universe (e.g. Risaliti et al. 1999). However, modelling of the hard X-ray background allows this ratio to be as high as 8:1 provided that half of the absorbed AGNs are Compton-thick (Gilli et al. 2007).

All the evidence points towards an obscured fraction that increases with decreasing AGN luminosity (which was noted a long time ago, see Lawrence \& Elvis 1982). Whether the obscured fraction evolves or not with redshift is still unclear (see e.g. Ueda et al. 2003; Treister \& Urry 2006; Gilli et al. 2007). The studies cited above range from $z \leq 0.3$ to $z \sim 2$, and luminous AGNs are present in the entire range (Zakamska et al. 2003; Lacy et al. 2007; Martínez-Sansigre et al. 2008). Lower-luminosity AGNs, however, are typically only found at low and intermediate redshifts $(z \lesssim 1)$, as expected from flux-limited samples. Thus, it is difficult at this stage to judge whether the obscured fraction varies with redshift.

\section{INFERRING $\langle\epsilon\rangle$}

Having discussed the radiated energy density from AGNs and the inferred ratio of obscured to unobscured sources, we proceed to use these to estimate the mean radiation efficiency of AGNs. We will inferr different values of $\langle\epsilon\rangle$, from the optical LF, with and without adding obscured AGNs, as well as the hard X-ray LF. This will illustrate the effect on $\langle\epsilon\rangle$ of the obscured AGN population.

Under the assumption that the local SMBHs grew by accretion:

$$
\rho_{\bullet, \text { loc }}=\rho_{\bullet, \text { acc }}
$$

With $\rho_{\bullet}$,acc given by Equations 3 or 4 , we can estimate the value of $\langle\epsilon\rangle$ which yields the closest agreement between $\rho_{\bullet}$,loc and $\rho_{\bullet}$,acc. For convenience we define $u \equiv U / C_{\nu}$, where $U$ is defined by Equation [5] or 6. This is done to keep separate and explicit the uncertainties in the LF (represented by $u$ ) and on the bolometric correction $\left(C_{\nu}\right)$. 
We now apply Bayes' theorem, to obtain the posterior PDF of $\langle\epsilon\rangle$, given the measurement of $\rho_{\bullet}$, loc in the local Universe and marginalising over the parameters $u,\langle R\rangle$ and $C_{\nu}$ :

$$
\begin{aligned}
& \mathrm{P}\left(\langle\epsilon\rangle \mid \rho_{\bullet}, \text { loc }\right)=\frac{\mathrm{P}(\langle\epsilon\rangle)}{\mathrm{P}\left(\rho_{\bullet}, \text { loc }\right)} \mathrm{P}\left(\rho_{\bullet}, \text { loc } \mid\langle\epsilon\rangle\right)= \\
& \frac{\mathrm{P}(\langle\epsilon\rangle)}{\mathrm{P}\left(\rho_{\bullet}, \text { loc }\right)} \int \mathrm{P}(u) \mathrm{d} u \times \\
& \int \mathrm{P}(\langle R\rangle) \mathrm{d}\langle R\rangle \int \mathrm{P}\left(C_{\nu}\right) \mathrm{P}\left(\rho_{\bullet} \text {, loc } \mid\langle\epsilon\rangle, u,\langle R\rangle, C_{\nu}\right) \mathrm{d} C_{\nu} .
\end{aligned}
$$

For our purpose, $\rho_{\bullet}$,loc is our data, so that $\mathrm{P}\left(\rho_{\bullet}\right.$,loc $)$ is the evidence, $\mathrm{P}(u), \mathrm{P}(\langle R\rangle)$ and $\mathrm{P}\left(C_{\nu}\right)$ are the prior PDFs for $u,\langle R\rangle$ and $C_{\nu}$ respectively. We have made use of the fact that our parameters are independent, so that $\mathrm{P}(\langle R\rangle \mid\langle\epsilon\rangle)=\mathrm{P}(\langle R\rangle), \mathrm{P}(u \mid\langle\epsilon\rangle)=\mathrm{P}(u)$ and $\mathrm{P}\left(C_{\nu} \mid\right.$ $\langle\epsilon\rangle)=\mathrm{P}\left(C_{\nu}\right) . \mathrm{P}\left(\rho_{\bullet}\right.$, loc $\left.\mid\langle\epsilon\rangle, u,\langle R\rangle, C_{\nu}\right)$ is the likelihood of $\rho_{\bullet}$, loc given $\langle\epsilon\rangle,\langle R\rangle$ and $C_{\nu}$ :

$\left.\mathrm{P}\left(\rho_{\bullet, \text { loc }} \mid\langle\epsilon\rangle, u,\langle R\rangle, C_{\nu}\right)=\frac{1}{(2 \pi)^{1 / 2} \sigma_{\rho_{\bullet}, \text { loc }}} e^{-\frac{1}{2}\left(\frac{\rho_{\bullet, \text { loc }}-\rho_{\bullet} \text {,acc }}{\sigma_{\bullet \bullet}, \text { loc }}\right.}\right)_{(9 .)}^{2}$

From Equation 4 and the definition of $u, \rho_{\bullet}$,acc is:

$$
\rho_{\bullet, \mathrm{acc}}=\frac{(1-\langle\epsilon\rangle)}{\langle\epsilon\rangle c^{2}} C_{\nu} u,
$$

or:

$$
\rho_{\bullet}, \mathrm{acc}=\frac{(1-\langle\epsilon\rangle)}{\langle\epsilon\rangle c^{2}}(1+\langle R\rangle) C_{\nu} u,
$$

depending on whether obscured AGNs are to be added on via the $(1+\langle R\rangle)$ term. Note that we have made the simplifying assumption that $\langle R\rangle$ is independent of luminosity, this will be addressed in Section 5.4

\subsection{Priors}

We now assign a prior PDF to each of the parameters $C_{\nu},\langle R\rangle, u$, and $\langle\epsilon\rangle$, considering three scenarios: a) considering only unobscured AGNs b) considering the X-ray absorbed and unabsorbed AGNs, but not the Comptonthick ones and c) attempting to include all obscured and unobscured AGNs d) same as c) but considering only the bright AGNs (quasars).

\subsection{1. $\mathrm{P}\left(C_{\nu}\right)$}

In this work we are interested in the bolometric output from accretion onto the SMBH, which we assume produces X-ray, optical and ultraviolet light predominantly and emits isotropically. We also assume that the dust responsible for the observed infrared emission (and the orientation-dependent SED) is on a larger scale, and not directly relevant to the accretion disk. Thus, the bolometric corrections for accretion onto the SMBH must convert from B-band luminosity to bolometric luminosity integrated over the optical, ultraviolet and X-ray range only.

We adopt the luminosity-dependent bolometric corrections of Marconi et al. (2004) and Hopkins et al. (2007), both of which follow these assumptions. We assume gaussian priors around these values, with the former having typical uncertainties $\frac{\sigma_{\mathrm{C}_{\mathrm{bol}}}}{C_{\mathrm{bol}}}=0.05$ and 0.10 for the $\mathrm{B}$-band and hard X-rays, respectively, and the latter $\frac{\sigma_{\mathrm{C}_{\mathrm{bol}}}}{C_{\mathrm{bol}}}=0.08$ and 0.11 for the same bands.

$$
\text { 5.1.2. } \mathrm{P}(u)
$$

We estimate the total radiated energy density from unobscured AGNs by using the B-band LF, and the total radiated energy density from unabsorbed and absorbed (but Compton-thin) AGNs using the hard X-ray LF. The evolution of the LF is such that the luminosity density at $z>5$ is similar to that at $z<1$, but the lookback time is much shorter, meaning the energy emitted at $z \geq 5$ is negligible. In other words, the integral in Equation 4 has converged by $z=5$. However, the LFs are not as well constrained in the range $3 \leq z \leq 5$, and the functional form chosen to represent the evolution (e.g. a 2nd or 3 rd order polynomial) can have unphysical effects. We therefore prefer to restrict the range over which the LFs are integrated to $0 \leq z \leq 3$. The missing radiated energy is expected to be $\$ 10 \%$ (see Section [3), and therefore negligible compared to our uncertainties.

Due to the fact that the bolometric corrections are luminosity-dependent and need to be included inside the integral when calculating $U$, we quote here the product of $u$ and $C_{\nu}$ assuming the mean value of $C_{\nu}$. The uncertainty quoted is only due to $u$, estimated by integrating different LFs, while the uncertainty in $C_{\nu}$ is encoded in the prior $\mathrm{P}\left(C_{\nu}\right)$.

For the B-band, due to the different survey areas and sensitivities, we use the LFs of Boyle et al. (2000) and Croom et al. (2004) at $z<1$ and of Wolf et al. (2003) in the range $1 \leq z \leq 3$ (using both pure density and pure luminosity evolution). The LF is integrated for all magnitudes brighter than $M_{\mathrm{B}}<-18\left(L_{\nu \mathrm{B}} \geq 8 \times 10^{20}\right.$ $\mathrm{W} \mathrm{Hz}{ }^{-1}$ ). The estimated total energy from the B-band LFs is found to be $u C_{\nu}=(1.1 \pm 0.2) \times 10^{51} \mathrm{~J} \mathrm{Mpc}^{-3}$, using the bolometric corrections of Marconi et al. (2004), and $u C_{\nu}=(1.6 \pm 0.4) \times 10^{51} \mathrm{~J} \mathrm{Mpc}^{-3}$ when using $C_{\nu}$ from Hopkins et al. (2007).

If only the most powerful AGNs are considered (quasars, brighter than $M_{\mathrm{B}}=-23$ or $L_{\nu \mathrm{B}}=7 \times 10^{22}$ $\mathrm{W} \mathrm{Hz}-1)$, we find $u C_{\nu}=(0.9 \pm 0.2) \times 10^{51} \mathrm{~J} \mathrm{Mpc}^{-3}\left(C_{\nu}\right.$ from Marconi et al. 2004) and $u C_{\nu}=(1.4 \pm 0.3) \times 10^{51}$ $\mathrm{J} \mathrm{Mpc}^{-3}\left(C_{\nu}\right.$ from Hopkins et al. 2007).

We note all of the B-band LFs are likely to be missing the population of reddened AGNs. These sources suffer from moderate amounts of extinction in the visual band, $A_{\mathrm{V}} \sim 1-5$, which suppresses the AGN signatures at optical but not at near-infrared wavelengths $(\sim 1-2 \mu \mathrm{m}$, energies $\sim 0.5-1 \mathrm{eV}$ ). Brown et al. (2006) have estimated these to comprise $\sim 20 \%$ of the unobscured population, so the above estimates of the energy density include this uncertainty.

For the hard X-ray LF, we use the LFs of Ueda et al. (2003) and Silverman et al. (2008). The LF is then integrated over $L_{\mathrm{X}} \geq 3 \times 10^{34} \mathrm{~W}$. The estimated radiated energy density, which includes Compton-thin absorbed AGNs, is then $u C_{\nu}=(4.5 \pm 0.1) \times 10^{51} \mathrm{~J} \mathrm{Mpc}^{-3}$ (using $C_{\nu}$ from Marconi et al. 2004) and $u C_{\nu}=(6.4 \pm 0.1) \times 10^{51} \mathrm{~J} \mathrm{Mpc}^{-3}$ $\left(C_{\nu}\right.$ from Hopkins et al. 2007). 


\subsection{3. $\mathrm{P}(\langle R\rangle)$}

As we have discussed in Section 4, the value of $\langle R\rangle$ inferred amongst high-luminosity AGNs is $\sim 2$, while amongst low-luminosity AGNs it is $\sim 4$. In Equations 4 and 11, we have made the simplification of keeping $\langle R\rangle$ independent of luminosity and redshift (and hence outside the integral). The question of whether $\langle R\rangle$ evolves or not with $z$ is still unclear but it seems likely that it does vary with luminosity (see Section 4).

We will repeat our analysis using priors centred on $\langle R\rangle \sim 2$ and 4 , to show the different values inferred for $\langle\epsilon\rangle$. In addition we will also carry out the analysis without including obscured AGNs, using Equations 3 and 10 . which is equivalent to using a prior with $\langle R\rangle=0$.

In the case of $\langle R\rangle \sim 2$, inferred from the bright AGNs, we assign a prior based on the estimate of Martínez-Sansigre et al. (2008). This can be approximated to a gaussian PDF with $\mu_{\langle R\rangle}=1.7$ and $\sigma_{R}=1.2$ (their large uncertainty arising from small number statistics).

Amongst low-luminosity AGNs, several studies suggest $\langle R\rangle \sim 4$, but there is no explicit estimate that we can use as a prior. For this case, we use a $\delta$-function prior centred on 4 . Due to the lack of scatter in $\langle R\rangle$, the posterior $\mathrm{PDF}$ for $\langle R\rangle=4$ obtained from the $\delta$-function prior will be artificially narrower than the posterior PDF assuming $\mu_{\langle R\rangle}=1.7$ and $\sigma_{R}=1.2$. This will be dealt with in Section 5.4.

The hard X-ray LF includes unabsorbed and Comptonthin absorbed AGN, so that only Compton-thick AGNs should be missing. The luminosity-dependent absorbed AGN fraction is also encoded in the LF. We do not attempt to correct for the missing Compton-thin population, so we are again using Equation 3 and 10 instead of 4 and 11 .

\subsection{4. $\mathrm{P}(\langle\epsilon\rangle)$}

For the efficiency, we reflect our ignorance by setting a prior in log space, $\mathrm{P}\left(\log _{10}[\langle\epsilon\rangle]\right)$, which is flat in the range $-3.0 \leq \log _{10}[\langle\epsilon\rangle] \leq 0.0$.

\subsection{The local density of $S M B H s$}

For $\rho_{\bullet, \text { loc }}$, we assume a value of $4.6_{-1.4}^{+1.9} \times 10^{5} \mathrm{M}_{\odot}$ $\mathrm{Mpc}^{-3}$, following Marconi et al. (2004). The likelihood is then assumed to be a gaussian distribution around this value (Equation 9). We compare the total $\rho_{\bullet}$, loc to the energy density computed using all AGNs brighter than $M_{\mathrm{B}}<-18$ (for the B-band LF) or $L_{\mathrm{X}} \geq 3 \times 10^{34} \mathrm{~W}$ (hard X-ray LF).

For the case of $\langle R\rangle \sim 2$, given that this value is motivated by luminous obscured AGNs (quasars), we also consider only the bright end of the optical LF (AGNs with $\left.M_{\mathrm{B}}<-23\right)$. We make the assumption that they are hosted by the progenitors of present-day elliptical galaxies, so we compare their integrated energy to the SMBH density in elliptical galaxies only. Marconi et al. (2004) estimated the SMBH density in elliptical galaxies to be $70 \%$ of the total SMBH density, or $3.2_{-1.0}^{+1.3} \times 10^{5} \mathrm{M}_{\odot}$ $\mathrm{Mpc}^{-3}$.

\subsection{The posterior PDFs for $\langle\epsilon\rangle$}

Figure 2 (panels a, b, c and d) shows the resulting posterior PDFs for $\log _{10}[\langle\epsilon\rangle]$, given our parametrisation, given our priors and given our assumed local density of SMBHs, $\rho_{\bullet}$, loc. Panel (a) shows the results from the B-band LFs without any correction for obscured AGNs $(\langle R\rangle=0)$, using our two sets of values for $C_{\nu}$.

Panel (b) shows the result of using the hard X-ray LF. The value of $u$ derived from the hard X-ray LF (Section 5.1.2) includes both X-ray unabsorbed AGNs and X-ray absorbed Compton-thin AGNs, but not absorbed Compton-thick AGNs.

Panel (c) shows the results from the B-band LFs and the two different bolometric corrections, with two different values of $\langle R\rangle \quad(\sim 2$ and 4$)$ used to correct for the obscured population (which includes the Compton-thick AGNs as well as the Compton-thin ones). Note that the posteriors for $\langle\epsilon\rangle$ derived from $\langle R\rangle=0$ and $\langle R\rangle=4$ are artificially narrower than that for $\langle R\rangle \sim 2$, due to the $\delta$ function priors for $\langle R\rangle$. This is dealt with in Section 5.4

Panel d) shows the posterior PDFs for the quasars. It is derived from the B-band LFs, integrating only over AGNs brighter than $M_{\mathrm{B}}=-23$, considering only $\langle R\rangle \sim 2$, and comparing to the SMBH density in local elliptical galaxies.

\subsection{Combining different estimates for $\langle\epsilon\rangle$}

The estimates for $\langle\epsilon\rangle$ shown in Figure 2 have been derived using different values for $C_{\nu}$, and in the case of pannel c, different values for $\langle R\rangle$. We now wish to combine the estimates in each panel, to obtain our four estimates for $\langle\epsilon\rangle$ for each of the three scenarios: a) considering only unobscured quasars from the B-band LF b) considering the absorbed and unabsorbed AGNs from the X-ray LF, but not the Compton-thick ones and c) attempting to include all obscured and unobscured AGNs by using the B-band LF and correcting by $(1+\langle R\rangle)$ finally $\mathrm{d})$ is the same as c) but only for the quasars.

In all four scenarios, we have used two different sets of bolometric corrections. We have no strong reason to believe one set is more appropriate than the other, but they do yield different values of $\langle\epsilon\rangle$. Additionally, in scenario $c$, have derived estimates for $\langle\epsilon\rangle$ in the presence of obscured AGNs based on two different priors for $\langle R\rangle$, one where $\langle R\rangle \sim 2$, the other with $\langle R\rangle=4$. Both of these values for $\langle R\rangle$ are believed to be accurate, but only appropriate at a certain luminosity. Instead, we have used a fixed value of $\langle R\rangle$ for the entire LF. We now wish to improve our estimate, by considering how appropriate each of our estimates is, and combining them.

We follow the arguments of Press (1997), and consider our different estimates of $\langle\epsilon\rangle$ from different combinations of the parameters $C_{\nu}$ and $\langle R\rangle$ as indepedent measurements. Although these inferred values of $\langle\epsilon\rangle$ have been derived using the same method, and are therefore apparently not independent, the reason they differ is due to the different assumptions for $C_{\nu}$ and $\langle R\rangle$, which are themselves independent. What we are doing here is to estimate the most likely value of $\langle\epsilon\rangle$, given the independent parameters assumed.

We must now assess the appropriateness of each estimate for $\langle\epsilon\rangle$ resulting from using each combination of parameters. Each resulting estimate of $\langle\epsilon\rangle,\langle\epsilon\rangle_{i}$, will be considered a good estimate or a bad estimate, depending on whether the values of $C_{\nu}$ and $\langle R\rangle$ assumed were appropriate or not. Bad estimates of $\langle\epsilon\rangle$ are simply those where the standard deviation has been underestimated. 
To each measurement $\langle\epsilon\rangle_{i}$, we assign the probability $p_{i}$ that the choice of parameters was the most appropriate and probability $\left(1-p_{i}\right)$ that it was not the most appropriate. We here consider all $p_{i}$ 's equal, simplifying to $p$.

The posterior probability for the efficiency $\langle\epsilon\rangle$, given data $D$, can then be written as

$$
\begin{aligned}
& \mathrm{P}\left(\log _{10}[\langle\epsilon\rangle] \mid \mathrm{D}\right)= \\
& \frac{\mathrm{P}\left(\log _{10}[\langle\epsilon\rangle]\right)}{\mathrm{P}(\mathrm{D})} \int \mathrm{P}(p) \prod_{i}\left[p \mathrm{P}_{G i}+(1-p) \mathrm{P}_{B i}\right] \mathrm{d} p
\end{aligned}
$$

where $\mathrm{P}_{G i}$ and $\mathrm{P}_{B i}$ are the probability distribution functions for good and bad measurements, derived from appropriate and inappropriate parameter values (see Press 1997, for a derivation of this result).

We marginalise over $p$. Thus, even if we do not have any information on the probability of a term being correct or not, and have no objective way of assigning values of $p$, we can still proceed. We simply integrate over the entire range of values for $p$, weighted by the prior $\mathrm{P}(p)$.

To estimate $\mathrm{P}\left(\log _{10}[\langle\epsilon\rangle] \mid \mathrm{D}\right)$, the posterior $\mathrm{PDF}$ for $\langle\epsilon\rangle$ given our different measurements, we assume a flat prior for $\log _{10}[\langle\epsilon\rangle] . \mathrm{P}\left(\log _{10}[\langle\epsilon\rangle]\right) / \mathrm{P}(\mathrm{D})$ is in practice simply a normalisation term. For $p$, we assign a prior flat in the range $0 \leq p \leq 1$, reflecting complete ignorance.

The good probability distribution functions $\mathrm{P}_{G i}$ are those where the uncertainty is correctly estimated, so we use our individual posteriors for each combination of parameters (the dashed, dotted or dash-dotted curves in Figure 2 panel a, b, c or d). For the bad PDFs, $\mathrm{P}_{B i}$, we choose to assume they would have been correct if they had quoted an uncertainty twice as large. We rederive the curves, using exactly the same procedure and priors of Section [5] but with a final uncertainty which is made artificially to be twice as large.

When combining the different estimates, we obtain an improved one that takes into account not only the different mean parameter values, but also the different uncertainties. For example, the potentially incorrect (underestimated) width of the estimates for $\langle\epsilon\rangle$ derived from a $\delta$-function prior at $\langle R\rangle=4$ has been dealt with.

The resulting combined posterior PDFs for $\langle\epsilon\rangle$ can be seen as the solid curves in Figure 2 a, b, c and d. These are our best estimate for the mean radiation efficiency, $\langle\epsilon\rangle$, given each scenario.

\subsection{Results}

Our results for the different scenarios illustrate how the efficiency estimated from the Soltan argument varies as the population of obscured AGNs is taken into account. In all panels of Figure 2, the solid lines show the result from combining the individual posterior PDFs assuming different parameters. The uncertainties quoted below correspond to the gaussian approximation of the solid lines in each figure. This gaussian approximation is extremely accurate for the low-efficiency tail of the PDFs, but underestimates the high-efficiency tail typically above the full-width half-maximum.

When we consider only the unobscured AGNs, we find $\log _{10}[\langle\epsilon\rangle]=-1.77_{-0.11}^{+0.16}$ (Figure 2a) or a relatively low efficiency, $\langle\epsilon\rangle=1.7_{-0.4}^{+0.8 \%}$. This is the efficiency derived when no obscured AGNs are taken into account.
Using the hard X-ray LF (Figure 2b), we find $\log _{10}[\langle\epsilon\rangle]=-1.20_{-0.10}^{+0.15}$ or $\langle\epsilon\rangle=6.4_{-1.3}^{+2.6} \%$. This estimate for $\langle\epsilon\rangle$ includes the absorbed Compton-thin population, but is still missing the Compton-thick AGNs.

Figure 2c shows the result if we integrate over the optical LF and consider the values of $\langle R\rangle$ for high-luminosity and low-luminosity AGNs, $\log _{10}[\langle\epsilon\rangle]=-$ $1.17_{-0.08}^{+0.11}$ or $\langle\epsilon\rangle=6.7_{-1.1}^{+1.9} \%$. We consider this our overall best estimate for $\langle\epsilon\rangle$, because it is expected to include the Compton-thick AGNs and therefore to be the least incomplete in terms of obscured AGNs.

Finally, when only obscured and unobscured quasars are considered we obtain the solid curve in Figure 2d. Here we are using the optical LF integrated above $M_{\mathrm{B}}=$ -23 and assuming $\langle R\rangle \sim 2$. We are also only comparing the radiated energy density to the mass density of local SMBHs in elliptical galaxies. The results can then be approximated as $\log _{10}[\langle\epsilon\rangle]=-1.27_{-0.11}^{+0.15}$ or $\langle\epsilon\rangle=5.4_{-1.2}^{+2.2} \%$.

The difference in efficiency between the B-band LF with $\langle R\rangle=0$ and the other results shows the cosmological importance of obscured (or absorbed) AGNs. In Section 6, we discuss how this affects the estimate of SMBH spins, under a set of assumptions. However, from the different results obtained using different values of $C_{\nu}$, visible in the different panels of Figure 2, we can also infer that the bolometric correction is still a critical source of uncertainty.

The value of $\langle\epsilon\rangle$ has been a matter of some debate, particularly whether its value is above or below $\sim 10 \%$. In Section 5.6 we compare to results obtained by other studies. We note that, from our best-estimate PDFs from Figure $2 \mathrm{~b}$, c and $\mathrm{d}$, we find $\mathrm{P}(\langle\epsilon\rangle \leq 10 \%)$ ranges between 79 and $88 \%$. Hence, our results strongly favour values of $\langle\epsilon\rangle$ below $10 \%$.

\subsection{Comparison to previous estimates}

Given the large uncertainties in $\langle R\rangle$, in $C_{\nu}$ and in $\rho_{\bullet}$, loc, our integrated approach is appropriate. Using this method, we obtain $\langle\epsilon\rangle \sim 7 \%$, and find our results consistent with those of Marconi et al. (2004), Shankar et al. (2004) and Merloni \& Heinz (2008) who used differential LFs (typically hard X-ray LFs) and local SMBH mass functions, and applied extra corrections to account for the Compton-thick population.

Our results are partially in agreement with those of Cao \& Li (2008), who find $\langle\epsilon\rangle \sim 8 \%$ when considering the AGNs leading to SMBHs with masses $<10^{8} \mathrm{M}_{\odot}$. However, they also find $\langle\epsilon\rangle \gtrsim 18 \%$ for $>10^{9} \mathrm{M}_{\odot}$, whereas we have found that $\langle\epsilon\rangle \sim 5 \%$ when comparing quasars to the SMBH mass density in elliptical galaxies. The SMBHs in ellipticals do not necessarily map one-to-one with the SMBHs with $>10^{9} \mathrm{M}_{\odot}$ ( see e.g. Marconi et al. 2004), but our results suggest that the mean radiation efficiency remains approximately constant.

We do not, however, find a good agreement with the results of $\mathrm{Yu} \& \mathrm{Lu}(2008)$, who derive a value of $\langle\epsilon\rangle \sim 16 \%$, or those of Wang et al. (2006) who used a completely independent approach to estimate $\langle\epsilon\rangle=20-35 \%$.

Surprisingly, our result is also consistent with that of Yu \& Tremaine (2002), despite the fact that they did not include the obscured AGN population. These authors used a lower value for $\rho_{\bullet}$,loc $\left(2.5 \pm 0.4 \times 10^{5} \mathrm{M}_{\odot} \mathrm{Mpc}^{-3}\right)$ and also used a higher value of $C_{\nu}$, since they did not re- 
move the infrared component of the quasar SED. These factors approximately cancel the effect of including the $(1+\langle R\rangle)$ term, explaining why our estimate of $\langle\epsilon\rangle$ is consistent with that of $\mathrm{Yu} \&$ Tremaine (2002).

Using the intensity of the X-ray background, and assuming a typical redshift of 2 for the sources dominating it, Elvis et al. (2002) estimated $\langle\epsilon\rangle \gtrsim 15 \%$. X-ray selected AGNs, however, have been observed to lie predominantly at lower redshifts (e.g. Barger et al. 2003; Ueda et al. 2003). If a typical value of $z=0.7-1$ is assumed instead, then the value of $\langle\epsilon\rangle$ derived following the method of Elvis et al. (2002) is reduced to $\sim 8-10 \%$, in reasonable agreement with our best estimate.

\section{ESTIMATING THE MEAN SMBH SPIN}

The rotation of a black hole determines the radius of the innermost stable circular orbit (ISCO) and therefore its binding energy. The rotation is measured in terms of the parameter $a \equiv \frac{J}{c m_{\bullet}}$ (in units of $\frac{G m_{\bullet}}{c^{2}}$ ), where $J$ is the angular momentum, $m_{\bullet}$ is the mass of the SMBH, $G$ is the gravitational constant and $c$ is the speed of light. The accretion efficiency $(\eta$, equal to 1 minus the binding energy and shown in Figure (3) is therefore determined by $a$.

The standard model for a thin accretion disk assumes no stresses are present between the ISCO and the Schwartzschild radius and neglects the effects of radiation from matter falling through this section, as well as assuming that any heat dissipated in the disk is immediately radiated away (Novikov \& Thorne 1973). These assumptions then lead to $\epsilon=\eta(a)$, so that the mean radiation efficiency can be used to estimate the mean SMBH spin.

Relaxing these assumptions has two important consequences, $\epsilon$ is altered and, through accretion alone the $\mathrm{SMBH}$ might reach an equilibrium spin value lower than $a=\frac{G m \bullet}{c^{2}}$.

When radiation inside the ISCO is considered, the black hole captures preferentially photons with opposite angular momentum, which limits the final spin to $a=0.998 \frac{\mathrm{Gm}}{\mathrm{c}^{2}}$ (Godfrev 1970; Thorne 1974). Magnetic fields in the region inside the ISCO can transport angular momentum outwards (Blandford \& Znajek 1977), possibly further limiting the equilibrium value of $a$ (Gammie et al. 2004; Krolik et al. 2005, estimate a limit of $\left.\sim 0.9 \frac{G m_{\bullet}}{c^{2}}\right)$.

The assumption that $\epsilon=\eta$ requires that no torques are present within the ISCO, and that the matter "plunges" into the black hole too quickly for any radiation to be significant. In this case, no energy can be extracted once matter has crossed the ISCO. However, the infalling matter can exhert a torque through magnetic fields, so that work is still done on the disk even after crossing the ISCO. The dissipation of this work can then lead to a higher radiative efficiency (e.g. Beckwith et al. 2008; Noble et al. 2008).

In the following section, we discuss our estimates of $\langle a\rangle$ from $\langle\epsilon\rangle$ assuming $\epsilon=\eta$, but from the discussion above these values should be considered as upper limits, since $\epsilon$ is really expected to be $>\eta(a)$. For the case of SMBH and disk co-rotating with $a \sim 0.9 \frac{G m \bullet}{c^{2}}$, the ISCO is very close to the Schwartzschild radius, so that there is little time to radiate in the region inside of the ISCO. In addition, the gravitational redshift diminishes the energy of the emergent radiation. For this case, Noble et al. (2008) have found that $\epsilon \sim 1.06 \eta$, so the difference is negligible compared to our uncertainties. However, for lower values of $a$ the difference between $\epsilon$ and $\eta$ might be larger, even of order unity (Beckwith et al. 2008).

Our value of $\langle\epsilon\rangle$ derived in the absence of obscured AGNs $\left(\langle\epsilon\rangle=1.7_{-0.4}^{+0.8} \%\right.$ for $\left.\langle R\rangle=0\right)$ is consistent with SMBHs having no rotation during periods of accretion $(\langle\epsilon\rangle<5.7 \%$ so that $a=0)$. An important point is therefore that when obscured AGNs are not included, the derived efficiency suggests that $\mathrm{SMBH}$ s were not spinning during periods of accretion.

When we do include the obscured AGNs, we find that values of $\langle\epsilon\rangle>5.7 \%$ are likely, so that the possibility that the SMBHs are rotating must be considered.

Maximally rotating black holes have $a=\frac{G m_{\bullet}}{c^{2}}$ with $\eta=42 \%$ (Bardeen 1970), although in the standard accretion disk model they are only expected to reach $a=$ $0.998 \frac{\mathrm{Gm}}{\mathrm{c}^{2}}$ at most, corresponding to $\eta=30 \%$ (Thorne 1974). From our posterior PDFs, $\mathrm{P}(\langle\epsilon\rangle \geq 30 \%)$ ranges from $0.3 \%^{2}$ to $2 \%^{2}$. Thus, under our parametrisation, we can rule out that SMBHs are, on average, maximally rotating with $\geq 98 \%$ confidence. More recent work suggests the upper limit on $a$ might be lower, $\sim 0.9 \frac{G m \bullet}{r^{2}}$, and with a radiative efficiency $\sim 15 \%$ (Noble et al. 2008). Again, from our posterior PDFs, $\mathrm{P}(\langle\epsilon\rangle \geq 15 \%)$ ranges from $2 \%$ to $8 \%$, so we rule out $\langle a\rangle \geq 0.9 \frac{G m \bullet}{c^{2}}$ at the $\geq 92 \%$ confidence.

For spinning black holes, $\langle\eta\rangle$ will vary depending on whether the ISCO is co-rotating or counter-rotating with respect to the black hole spin (see Figure 3). To estimate the mean value of $a$ two possible limiting scenarios can be envisaged:

A) The SMBHs grow from the seed in one long episode of accretion. In this case one single accretion disk is expected to be able to provide enough mass to turn the seed into a SMBH. The angular momentum of the seed is negligible compared to that of the accretion disk, so the disk will inevitably force the black hole to co-align (Scheuer \& Feiler 1996; King et al. 2005). It is therefore safe to estimate the efficiency assuming the black hole and ISCO are always co-rotating. In this case, our best estimate of $\langle\epsilon\rangle=6.7_{-1.1}^{+1.9} \%$ corresponds to SMBHs rotating with $\langle a\rangle=0.25_{-0.25}^{+0.30} \frac{\mathrm{Gm} \bullet}{\mathrm{c}^{2}}$ during periods of accretion (Figure 3, solid line).

$B$ ) The $S M B H s$ grow in many shorter periods of accretion. The implication here is that the angular momenta of the accretion disk and black hole are comparable and are also initially randomly oriented with respect to each other. Depending on the initial configuration and ratio of angular momenta, the black hole and disk will either co-align or counter-align (King et al. 2005; Lodato \& Pringle 2006). It is expected that co- and counter-aligned accretion is equally probable, so when estimating $a$, a mean $\langle\eta(a)\rangle$ from co- and counter-rotating orbits should be used (the symmetrised curve suggested by King et al. 2008, shown in Figure 3 by the dashed line). In this case, for our best estimate of $\langle\epsilon\rangle$ we inferr $\langle a\rangle=0.59_{-0.59}^{+0.25} \frac{G m \bullet}{c^{2}}$.

2 These probabilities have been calculated from the actual PDFs, not the gaussian approximation which underestimates the probability in the tails of the PDFs. 
Mergers involving SMBHs are expected to prevent $a$ from reaching the highest values (see the work of Hughes \& Blandford 2003, who find half of their parameter space will lead to $\left.a \leq 0.4 \frac{G m \bullet}{c^{2}}\right)$. For both accretion scenarios, and for $a \lesssim 0.8 \frac{G m \bullet}{c^{2}}$, the dependence of $\eta$ on $a$ is weak so that it is difficult to constrain $\langle a\rangle$ from our estimate of $\langle\epsilon\rangle$. However, we have found that maximally rotating SMBHs are not favoured since $\mathrm{P}(\langle\epsilon\rangle \geq 30 \%) \leq 2 \%$, and our best estimates for $a$ are in reasonable agreement with the results of Hughes \& Blandford (2003).

Our estimates for $\langle a\rangle$ are nevertheless also consistent with the value of $a \geq 0.5 \frac{G m \bullet}{c^{2}}$ estimated for the SMBH in the Milky Way, Sagittarius A* Genzel et al. 2003, who explained the short near-infrared flare period of 17 minutes as being close the ISCO for co-rotating disk and $\mathrm{SMBH})$. A SMBH with $a \geq 0.5 \frac{G m_{\bullet}}{c^{2}}$ and a prograde orbit has an accretion efficiency $\eta \geq 8 \%$. Thus, the limit on $a$ inferred for the individual SMBH Sagittarius $A^{\star}$ is in good agreement with our best estimate of the mean radiation efficiency, $\langle\epsilon\rangle$, assuming the standard thin disk model and ignoring the magnetic fields.

Indeed, we remind the reader that we have estimated $\langle a\rangle$ assuming $\epsilon=\eta$, but at the beginning of this Section we have argued for $\epsilon>\eta$. Hence, our estimates of $\langle a\rangle$ can be considered upper limits, since for a given true value of $a, \epsilon>\eta(a)$ (Krolik et al. 2005; Noble et al. 2008; Beckwith et al. 2008). For $a \sim 0.9 \frac{G m_{\bullet}}{c^{2}}$, it seems that $\epsilon$ is only slightly larger than $\eta$, but for lower values of $a$ the difference is expected to be larger, since the time available for radiation is longer and the gravitational redshift is less severe. This only strengthens our main conclusion that our inferred value of $\langle\epsilon\rangle$ does not require accreting SMBHs to be rotating particularly rapidly, on average, during periods of accretion.

\section{THE MEAN E-FOLDING TIME}

Given our assumption that AGNs grow by accretion, their mass will grow exponentially provided that the luminosity is proportional to the mass. Thus, if AGNs radiate at a fraction $\lambda$ of their Eddington luminosity, then they will grow exponentially with an $e$-folding time:

$$
\tau=\frac{\epsilon}{\lambda(1-\epsilon)} \frac{c \sigma_{\mathrm{T}}}{4 \pi G m_{\mathrm{p}}}
$$

where $m_{\mathrm{p}}$ is the mass of the proton and $\sigma_{T}$ is the Thomson electron scattering cross-section. Our estimate of $\langle\epsilon\rangle$ is completely independent of $\lambda$, and it is only to estimate $\tau$ that we require an estimate of the Eddington ratio $\lambda$.

Estimates of $\lambda$ exist for unobscured quasars, derived from their bolometric luminosities and virial-mass estimates for the SMBH mass. McLure \& Dunlop (2004) found $\lambda \sim 0.15-0.5$ in the range $0.1 \leq z \leq 2.1$, with significant scatter. We approximate the distribu-

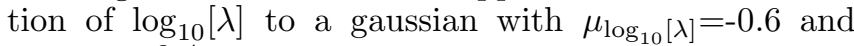
$\sigma_{\log _{10}(\lambda)}=0.4$.

When combined with our estimate of $\langle\epsilon\rangle$ for quasars $\left(5.4_{-1.2}^{+2.2} \%\right)$, this leads to an estimate of $\log _{10}[\langle\tau\rangle / \mathrm{Myr}]=$ $2.0 \pm 0.4$, or $\langle\tau\rangle=100_{-60}^{+151} \mathrm{Myr}$. This value is consistent with (although at the high end of) other current constraints from quasar clustering measurements, from arguments based on coevolution of quasars, SMBHs and host galaxies, from arguments of simultaneous trigger- ing of quasars and starbursts and from the lengths of radio jets. These estimates all suggest $\tau$ is in the range 10-100 Myr (see Martini 2004, for a review).

Such a long value of $\tau$ suggests SMBHs require a long time to build up their mass. At $z=6.4, \mathrm{SMBH}$ masses of $2-6 \times 10^{9} \mathrm{M}_{\odot}$ have been estimated (Willott et al. 2003; Barth et al. 2003), when the Universe was only 840 Myrs old. This suggests that the seeds that formed the $z=6$ quasars might have been as large as $\sim 10^{5}-10^{6} \mathrm{M}_{\odot}$.

The estimates of the SMBH mass and particularly our estimate of the $e$-folding time have very large uncertainties and the quasars at $z=6.4$ could have shorter $e$ folding times. They might be accreting closer to the Eddington limit than $\lambda \sim 0.25$, the mean of the $0.1 \leq z \leq 2.1$ quasars (McLure \& Dunlop 2004), or radiating less efficiently than $\sim 7 \%$. This would then imply significantly smaller seeds. For example, if $\tau \sim 60 \mathrm{Myr}$ (40 Myr), the seeds could be $\sim 10^{3} \mathrm{M}_{\odot}\left(\sim 1 \mathrm{M}_{\odot}\right)$.

\section{SUMMARY}

We have revisited the mean radiation efficiency from accretion onto SMBHs, $\langle\epsilon\rangle$, quantifying the effect introduced by taking into account a large population of obscured AGNs. We estimate the cumulative energy density of the AGN population from the unobscured AGN LF by applying appropriate bolometric corrections and correcting for the obscured population. The efficiency $\langle\epsilon\rangle$ is estimated by comparing this energy density to the SMBH mass density in the local Universe.

When no obscured AGNs are included, we find $\langle\epsilon\rangle=1.7_{-0.4}^{+0.8 \%}$ which does not require $\mathrm{SMBH}$ s to rotate at all during periods of accretion $(\langle a\rangle=0)$.

Using the hard X-ray LF, which includes absorbed Compton-thin AGNs, we find $\langle\epsilon\rangle=6.4_{-1.3}^{+2.6 \%}$. Using the B-band LFs, correcting for the obscured population (which includes the Compton-thick AGNs), we find $\langle\epsilon\rangle=6.7_{-1.1}^{+1.9} \%$, which we consider to be our best estimate.

We have also derived $\langle\epsilon\rangle$ for the powerful AGNs only (the quasars), and find $\langle\epsilon\rangle=5.4_{-1.2}^{+2.2} \%$.

Depending on whether the SMBHs and accretion disks are assumed to be always co-rotating, or instead to counter-rotate in half of the cases, we estimate the rotation parameter during periods of accretion to be $\langle a\rangle=$ $0.25_{-0.25}^{+0.30} \frac{G m_{\bullet}}{c^{2}}$ or $\langle a\rangle=0.59_{-0.59}^{+0.25} \frac{G m_{\bullet}}{c^{2}}$, respectively

Efficiencies $\geq 30 \%(\geq 15 \%)$ are ruled out at $\geq 98 \%$ $(\geq 92 \%)$ confidence, so the SMBHs are not, on average, maximally rotating during the periods of accretion. Efficiencies $\leq 10 \%$ are favoured, with $79 \%-88 \%$ of the probability density in this region.

Finally, combining our best estimate for $\langle\epsilon\rangle$ with estimates of the Eddington ratio for unobscured quasars yields an estimate of the $e$-folding time of $\langle\tau\rangle=$ $100_{-60}^{+151}$ Myr. This suggests that the seeds of $z=6$ quasars might have been very large, potentially as large as $\sim 10^{6} \mathrm{M}_{\odot}$. However, the large uncertainties in $\tau$ translate into order-of-magnitude uncertainties for the masses of the seeds, which could be as small as $\sim 1 \mathrm{M}_{\odot}$.

We are grateful to John Miller, Andrew King and Giuseppe Lodato for useful discussions, to Ross McLure for access to the distribution of Eddington ratios, to Fabio Fontanot and Mark Sargent for comments on the 
manuscript and to the anonymous referee for suggestions that improved the article significantly.

\section{REFERENCES}

Alonso-Herrero, A., et al. 2006, ApJ, 640, 167

Baker, J. C., Hunstead, R. W., Athreya, R. M., Barthel, P. D., de Silva, E., Lehnert, M. D., \& Saunders, R. D. E. 2002, ApJ, 568, 592

Bardeen, J. M. 1970, Nature, 226, 64

Barger, A. J., et al. 2003, AJ, 126, 632

Barth, A. J., Martini, P., Nelson, C. H., \& Ho, L. C. 2003, ApJ, 594, L95

Beckwith, K., Hawley, J. F., \& Krolik, J. H. 2008, MNRAS, 1003

Blandford, R. D. \& Znajek, R. L. 1977, MNRAS, 179, 433

Boyle, B. J., Shanks, T., Croom, S. M., Smith, R. J., Miller, L., Loaring, N., \& Heymans, C. 2000, MNRAS, 317, 1014

Brandt, W. N. \& Hasinger, G. 2005, ARA\&A, 43, 827

Brown, M. J. I. et al. 2006, ApJ, 638, 88

Cao, X. \& Li, F. 2008, ArXiv e-prints, 808

Croom, S. M., Smith, R. J., Boyle, B. J., Shanks, T., Miller, L., Outram, P. J., \& Loaring, N. S. 2004, MNRAS, 349, 1397

Donley, J. L., Rieke, G. H., Pérez-González, P. G., Rigby, J. R., \& Alonso-Herrero, A. 2007, ApJ, 660, 167

Donley, J. L., Rieke, G. H., Rigby, J. R., \& Pérez-González, P. G. 2005, ApJ, 634, 169

Eckart, A. \& Genzel, R. 1996, Nature, 383, 415

Elvis, M., Risaliti, G., \& Zamorani, G. 2002, ApJ, 565, L75

Gammie, C. F., Shapiro, S. L., \& McKinney, J. C. 2004, ApJ, 602, 312

Genzel, R., Schödel, R., Ott, T., Eckart, A., Alexander, T., Lacombe, F., Rouan, D., \& Aschenbach, B. 2003, Nature, 425, 934

Gilli, R., Comastri, A., \& Hasinger, G. 2007, A\&A, 463, 79

Godfrey, B. B. 1970, Phys. Rev. D, 1, 2721

Hopkins, P. F., Richards, G. T., \& Hernquist, L. 2007, ApJ, 654, 731

Hughes, S. A. \& Blandford, R. D. 2003, ApJ, 585, L101

Ivezić, Z., et al. 2002, AJ, 124, 2364

King, A. R., Lubow, S. H., Ogilvie, G. I., \& Pringle, J. E. 2005, MNRAS, 363, 49

King, A. R., Pringle, J. E., \& Hofmann, J. A. 2008, MNRAS, 385, 1621

Kormendy, J. \& Richstone, D. 1995, ARA\&A, 33, 581

Krolik, J. H., Hawley, J. F., \& Hirose, S. 2005, ApJ, 622, 1008

Lacy, M., Petric, A. O., Sajina, A., Canalizo, G., Storrie-Lombardi, L. J., Armus, L., Fadda, D., \& Marleau, F. R. 2007, AJ, 133, 186 Lawrence, A. \& Elvis, M. 1982, ApJ, 256, 410

Lodato, G. \& Pringle, J. E. 2006, MNRAS, 368, 1196

Lynden-Bell, D. 1969, Nature, 223, 690

Magorrian, J., et al. 1998, AJ, 115, 2285

Marconi, A., Risaliti, G., Gilli, R., Hunt, L. K., Maiolino, R., \& Salvati, M. 2004, MNRAS, 351, 169
Martínez-Sansigre, A., Lacy, M., Sajina, A., \& Rawlings, S. 2008, ApJ, 674, 676

Martínez-Sansigre, A., et al. 2007, MNRAS, 379, L6

Martínez-Sansigre, A., Rawlings, S., Lacy, M., Fadda, D., Jarvis, M. J., Marleau, F. R., Simpson, C., \& Willott, C. J. 2006, MNRAS, 370, 1479

Martini, P. 2004, in Coevolution of Black Holes and Galaxies, ed. L. C. Ho, 169-+

McLure, R. J. \& Dunlop, J. S. 2004, MNRAS, 352, 1390

Merloni, A. \& Heinz, S. 2008, MNRAS, 388, 1011

Noble, S. C., Krolik, J. H., \& Hawley, J. F. 2008, ArXiv e-prints, 808

Novikov, I. D. \& Thorne, K. S. 1973, in Black Holes (Les Astres Occlus), 343-450

Polletta, M. d. C. et al. 2006, ApJ, 642, 673

Press, W. H. 1997, in Unsolved Problems in Astrophysics, 49-60

Rees, M. J. 1984, ARA\&A, 22, 471

Reyes, R., et al. 2008, ArXiv e-prints, 801

Rigby, J. R., Rieke, G. H., Donley, J. L., Alonso-Herrero, A., \& Pérez-González, P. G. 2006, ApJ, 645, 115

Risaliti, G., Maiolino, R., \& Salvati, M. 1999, ApJ, 522, 157

Salpeter, E. E. 1964, ApJ, 140, 796

Scheuer, P. A. G. \& Feiler, R. 1996, MNRAS, 282, 291

Shankar, F., Salucci, P., Granato, G. L., De Zotti, G., \& Danese, L. 2004, MNRAS, 354, 1020

Silverman, J. D., et al. 2008, ApJ, 679, 118

Simpson, C. 2005, MNRAS, 360, 565

Soltan, A. 1982, MNRAS, 200, 115

Thorne, K. S. 1974, ApJ, 191, 507

Treister, E. \& Urry, C. M. 2006, ApJ, 652, L79

Ueda, Y., Akiyama, M., Ohta, K., \& Miyaji, T. 2003, ApJ, 598, 886

Wang, J.-M., Chen, Y.-M., Ho, L. C., \& McLure, R. J. 2006, ApJ, $642, \mathrm{~L} 111$

Willott, C. J., McLure, R. J., \& Jarvis, M. J. 2003, ApJ, 587, L15

Willott, C. J., Rawlings, S., Archibald, E. N., \& Dunlop, J. S. 2002, MNRAS, 331, 435

Willott, C. J., Rawlings, S., Blundell, K. M., \& Lacy, M. 2000, MNRAS, 316, 449

Wolf, C., Wisotzki, L., Borch, A., Dye, S., Kleinheinrich, M., \& Meisenheimer, K. 2003, A\&A, 408, 499

Yu, Q. \& Lu, Y. 2008, ArXiv e-prints, 808

Yu, Q. \& Tremaine, S. 2002, MNRAS, 335, 965

Zakamska, N. L., Strauss, M. A., Heckman, T. M., Ivezić, Ž., \& Krolik, J. H. 2004, AJ, 128, 1002

Zakamska, N. L., et al. 2003, AJ, 126, 2125 

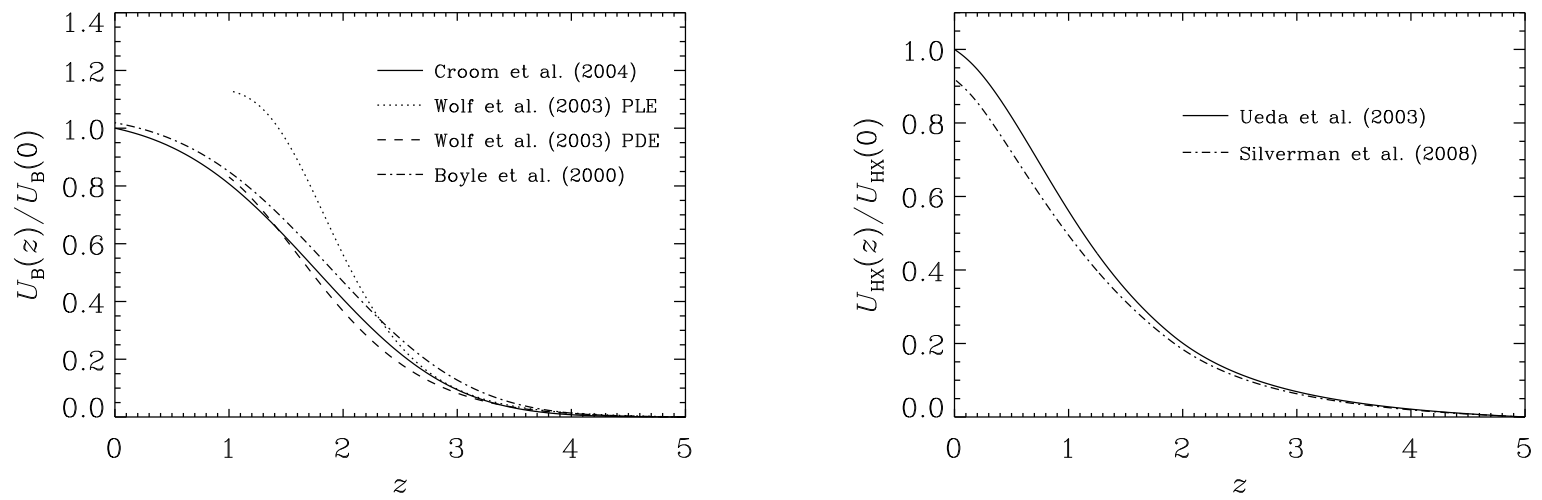

FIG. 1. - Cumulative energy densities for the optical LFs (left) and hard X-ray LF (right). We remove any dependence on $C_{\nu}$ by dividing them by the maximum value (at $z=0$ ). The maximum values are taken from the Croom et al. (2004) LF (left) and from the Ueda et al. (2003) LF (right). The Wolf et al. (2003) LF, defined only for $z \geq 1$, agrees well with the other two LFs assuming pure density evolution (PDE). However, pure luminosity evolution (PLE) predicts a somewhat larger value for $U_{\mathrm{B}}$. The optical LFs include only unobscured AGNs, while the X-ray LF also includes Compton-thin absorbed AGNs.
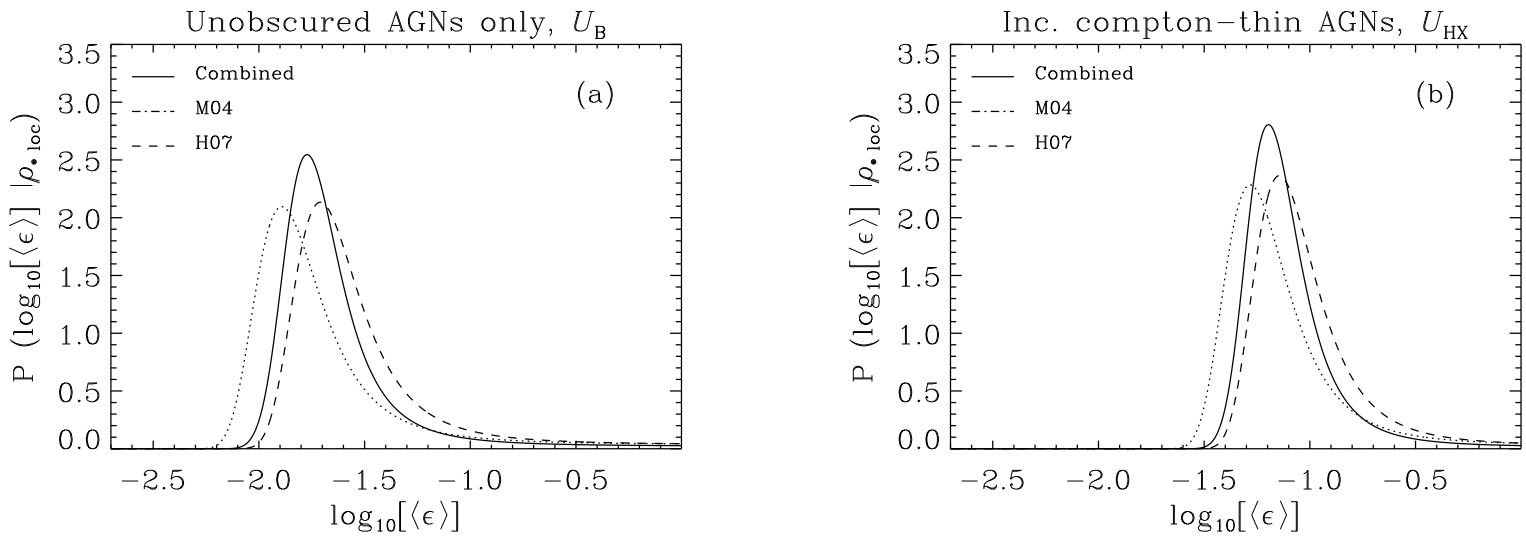

FIG. 2.- Posterior PDF for $\langle\epsilon\rangle$ derived from the optical LFs for unobscured AGNs only (a) and using the hard X-ray LF, with no correction for Compton-thick absorbed AGNs (b). The posteriors derived from the bolometric corrections of Marconi et al. (2004) are marked as 'M04', whereas those from Hopkins et al. (2007) are marked as 'H07'. The combined posteriors, derived in Section 5.4 are shown as the solid curve. For panel (a), the optical LF is integrated over all magnitudes brighter than $M_{\mathrm{B}}=-18\left(L_{\nu \mathrm{B}} \geq 8 \times 10^{20} \mathrm{~W} \mathrm{~Hz}^{-1}\right)$, whereas for panel (b) the LF has been integrated over all $L_{\mathrm{X}} \geq 3 \times 10^{34} \mathrm{~W}$.
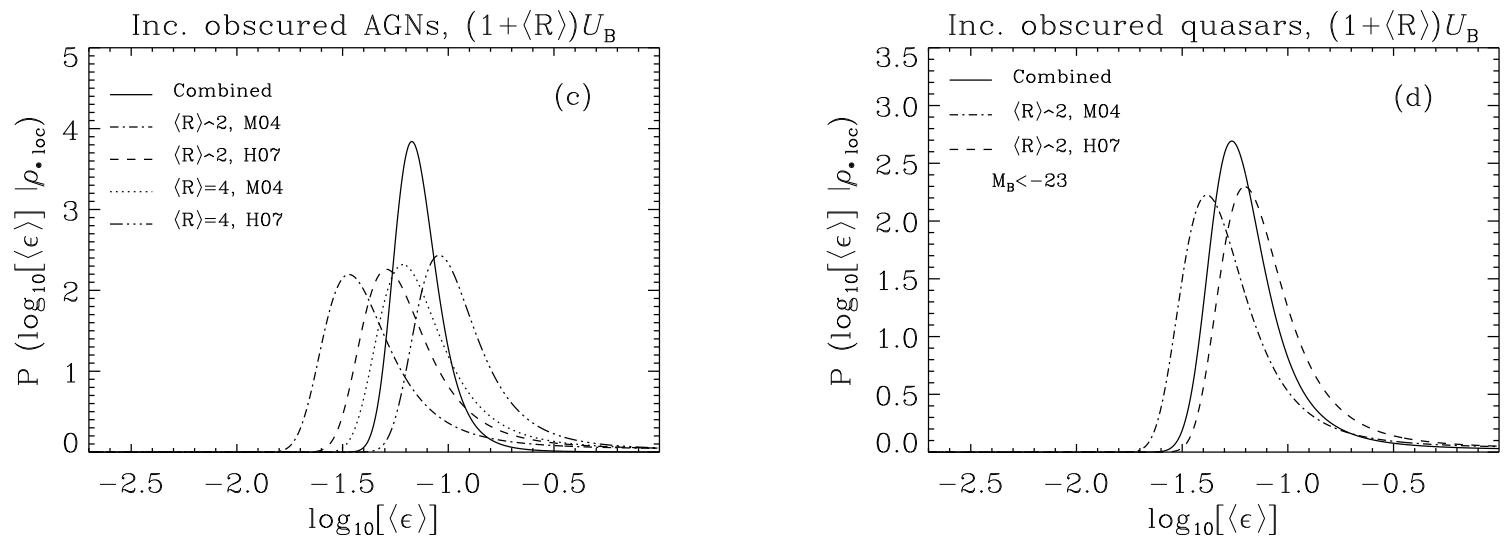

FIG. 2.- Continued. Posterior PDFs for $\langle\epsilon\rangle$ derived from the optical LFs correcting for the obscured AGNs using $\langle R\rangle$. In panel (c), the optical LF is integrated over all magnitudes brighter than $M_{\mathrm{B}}=-18$, and two different values of $\langle R\rangle$ are used $(\langle R\rangle \sim 2$ and $=4)$. We consider this our best estimate for $\langle\epsilon\rangle$. In panel (d), the LF has been integrated over all magnitudes brighter than $M_{\mathrm{B}}=-23$, and only $\langle R\rangle \sim 2$ is used, to estimate the value of $\langle\epsilon\rangle$ from the bright AGN population (the quasars). 


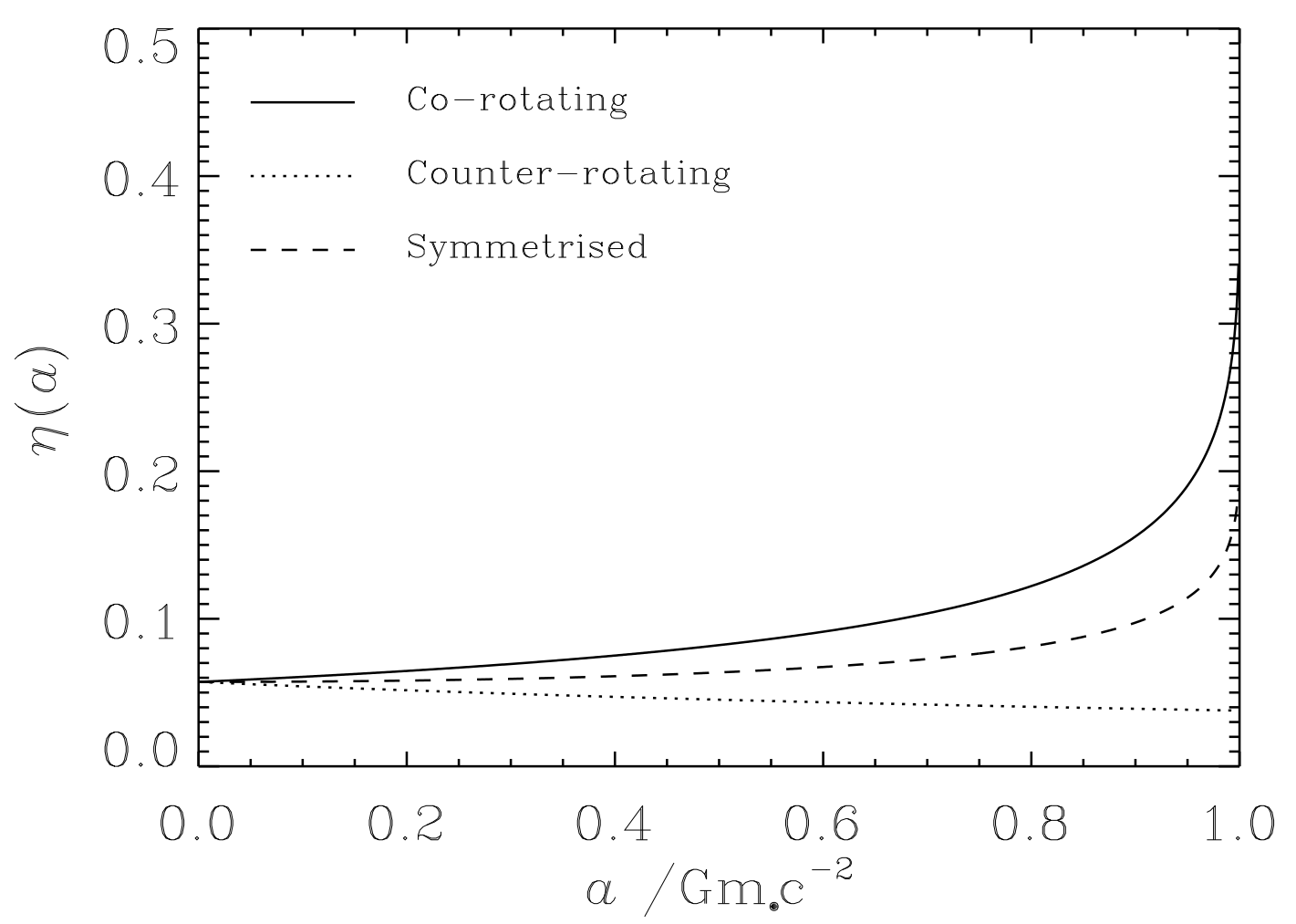

FIG. 3.- Accretion efficiency $\eta$ as a function of rotation parameter $a$. The solid curve represents the case of the SMBH co-rotating with the inner part of the accretion disk, so the innermost stable circular stable orbit (ISCO) is prograde. The dotted line represents the case of counter-rotating SMBH and inner accretion disk (the ISCO is retrograde). The dashed curve represents the mean of both curves (the "symmetrised" curve, see King et al. 2008). 